\title{
IMPACTO DA SUPERVISÃO CLÍNICA EM ENFERMAGEM NA AVALIAÇÃO E INTERVENÇÃO NO AUTOCUIDADO
}

\author{
IMPACT OF CLINICAL SUPERVISION IN NURSING ON \\ SELF-CARE EVALUATION AND INTERVENTION
}

\section{IMPACTO DE LA SUPERVISIÓN CLÍNICA DE ENFERMERÍA EN LA EVALUACIÓN E INTERVENCIÓN DEL AUTOCUIDADO}

\author{
Inês Rocha ${ }^{1}$ \\ António Luís Carvalho ${ }^{2}$ \\ Cristina Barroso Pinto ${ }^{3}$ \\ Agostinho Rodrigues ${ }^{4}$ \\ Vânia Rochas
}

Como citar este artigo: Rocha I, Carvalho AL, Pinto CB, Rodrigues A, Rocha V. Impacto da supervisão clínica em enfermagem na avaliação e intervenção no autocuidado. Rev baiana enferm. 2021;35:e43356.

Objetivo: avaliar concordância, relacionada ao grau de dependência no autocuidado, entre pesquisador, enfermeiros e prontuários antes e após implementação do Modelo SafeCare e avaliar as intervenções de enfermagem relativas ao autocuidado prescritas, antes e após implementação do Modelo SafeCare. Método: estudo quase experimental. Para coleta dos dados, em 2017 e 2019, utilizou-se instrumento de avaliação do autocuidado antes e após implementação do Modelo de supervisão clínica em enfermagem (SafeCare). Resultados: participaram 216 pacientes. A concordância entre pesquisador e enfermeiros aumentou do pré-teste para o pós-teste nos autocuidados higiene $(\mathrm{k}=0,79)$, alimentar-se $(\mathrm{k}=0,73)$ e posicionar-se $(\mathrm{k}=0,79)$. Nas intervenções de enfermagem, verificou-se existência de concordância entre todos os avaliadores no pós-teste, ao contrário do pré-teste em que não existiu concordância na intervenção "incentivar o autocuidado: higiene" entre pesquisador e enfermeiros/prontuários. Conclusão: a concordância entre formulários preenchidos pelos grupos individualmente aumentou significativamente na maioria dos autocuidados avaliados e das intervenções prescritas após implementação do Modelo SafeCare.

Descritores: Enfermagem. Supervisão de Enfermagem. Autocuidado. Diagnóstico de Enfermagem. Qualidade da Assistência à Saúde.

Objective: to assess the agreement between researcher, nurses and medical records in relation to self-care dependency levels before and after the implementation of the SafeCare Model and to evaluate the nursing interventions related to selfcare provided before and after the implementation of the SafeCare Model. Method: quasi-experimental study. For data collection, in 2017 and 2019, a self-care assessment instrument was used before and after the implementation of the model of clinical supervision in nursing (SafeCare). Results: 216 patients participated in the study. Agreement between researcher and nurses increased from pre-test to post-test in bygiene ( $k=0.79)$, self-feeding $(k=0.73)$ and self-transferring $(k=0.79)$. In nursing interventions, there was agreement between all evaluators in the post-test, unlike the pre-test, when there was no agreement between researcher and nurses/medical records in the intervention "promoting self-care: bygiene. Conclusion: the agreement between forms filled out individually by the groups increased significantly in most of the self-care measures assessed and, in the interventions, provided after the implementation of the SafeCare Model.

Descriptors: Nursing. Nursing, Supervisory. Self Care. Nursing Diagnosis. Quality of Health Care.

\footnotetext{
Enfermeira. Mestre em Supervisão Clínica em Enfermagem. Instituto de Ciências Biomédicas de Abel Salazar da Universidade do Porto. Porto, Portugal. inesarsrocha@gmail.com. http://orcid.org/0000-0002-3036-0002.

Enfermeiro. Doutor em Enfermagem. Professor Coordenador da Escola Superior de Enfermagem do Porto. Porto, Portugal. http://orcid.org/0000-0003-10 I 7-4787.

Enfermeira. Doutora em Enfermagem. Professora Adjunta da Escola Superior de Enfermagem do Porto. Porto, Portugal. http://orcid.org/0000-0002-6077-4I 50.

Enfermeiro. Escola Superior de Enfermagem do Porto. Porto, Portugal. http://orcid.org/0000-0003-2638-0278.

Psicóloga. Doutora em Psicologia. Membro Integrado do Centro de Psicologia da Universidade do Porto. Porto, Portugal. http://orcid.org/0000-0002-6251 -6809.
} 
Objetivo: analizar la concordancia relacionada con el grado de dependencia en el autocuidado, entre el investigador, las enfermeras y las historias clinicas antes y después de la implantación del Modelo SafeCare y evaluar las intervenciones de enfermería con respecto al autocuidado, prescritas antes y después de la implantación del Modelo SafeCare. Método: estudio cuasi-experimental, en el que se utilizó, para la recolección de datos entre 2017 y 2019, un instrumento para evaluar el autocuidado antes y después de la implementación del Modelo de supervisión clinica en enfermería (SafeCare). Resultados: participaron 216 pacientes. La concordancia entre el investigadory las enfermeras aumentó desde la prueba previa a la prueba posterior en los cuidados personales: higienizarse $(k=0,79)$, alimentarse $(k=0,73)$ y posicionarse $(k=0,79)$. En las intervenciones de enfermería, hubo acuerdo entre todos los calificadores en la prueba posterior, a diferencia de la prueba previa, en la que no bubo acuerdo en la intervención "fomento del autocuidado: higienización" entre investigador y enfermeros/registros. Conclusión: la concordancia entre los formularios rellenados por los grupos de forma individual aumentó significativamente en la mayoría de los autocuidados evaluados y en las intervenciones prescriptas tras la implementación del Modelo SafeCare.

Descriptores: Enfermería. Supervisión de Enfermería. Autocuidado. Diagnóstico de Enfermería. Calidad de la Atención a la Salud.

\section{Introdução}

A supervisão clínica em Enfermagem é considerada fundamental para uma prática profissional de qualidade ${ }^{(1-2)}$. Pode ser definida como um acompanhamento formal que, por meio da reflexão e da análise da prática clínica, tem como objetivo a promoção da tomada de decisão autónoma dos enfermeiros, de forma a potenciar a segurança dos cuidados e a proteção dos pacientes ${ }^{(3)}$.

A supervisão clínica promove maior consciência da responsabilidade profissional, favorecendo o desenvolvimento profissional e uma prática baseada na evidência, refletindo-se, a nível institucional, num ambiente propício ao bem-estar dos profissionais e numa redução dos incidentes e das reclamações ${ }^{(2,4)}$. Assim, pelas vantagens que a supervisão clínica origina, justifica-se a sua implementação e manutenção ${ }^{(5)}$.

Existem vários modelos de supervisão clínica em enfermagem, mas, até ao momento, todos se têm deparado com a dificuldade em dar resposta à diversidade dos contextos da prática e ao potencial das várias equipes de enfermagem ${ }^{(6)}$. Além disso, por norma, não apresentam a sua operacionalização quanto aos participantes, quantidade e conteúdos de supervisão clínica administrados, o que inviabiliza a sua replicação a posteriori $i^{(7)}$.

O Modelo SafeCare, criado por um grupo de investigadores portugueses do Projeto SafeCare, surgiu como resposta a essas lacunas do conhecimento. Tem como finalidade contribuir para a promoção da segurança e da qualidade dos cuidados de enfermagem, assim como desenvolver, nos enfermeiros, um conjunto de competências que lhes permitam desempenhar um exercício profissional de excelência, identificando áreas de intervenção de forma a que a sua implementação possa vir a ser efetuada nos diversos contextos dos cuidados ${ }^{(4)}$. Além disso, o modelo também busca contribuir na formulação de respostas às necessidades atuais colocadas pela lógica instalada nos serviços, como, por exemplo, aquelas decorrentes da implementação de sistemas de qualidade ${ }^{(6)}$. Importa ainda salientar que existe escassez de estudos de supervisão clínica que contemplem uma metodologia quantitativa $^{(2)}$. O Modelo SafeCare, pelo fato de os instrumentos utilizados na sua implementação serem de carácter quantitativo, vai de encontro ao que a evidência sugere.

Assim, o Modelo SafeCare comporta quatro etapas: diagnóstico de situação; identificação das necessidades em supervisão clínica; implementação do Modelo SafeCare; e avaliação dos resultados.

Na primeira etapa (diagnóstico de situação), tendo em consideração as necessidades visíveis no contexto de cuidados, definem-se, conjuntamente com os enfermeiros gestores (enfermeiro diretor, enfermeiro supervisor dos serviços e enfermeiros chefes), as variáveis a serem estudadas. 
Nesta etapa, também se aplica o instrumento de avaliação do autocuidado.

$\mathrm{Na}$ segunda etapa (identificação das necessidades em supervisão clínica), realizam-se reuniões com os enfermeiros para identificar quais os aspetos, na sua opinião, que necessitam de mudança: podem ser intrínsecos, de índole pessoal, ou extrínsecos, relativos à organização do serviço e prestação de cuidados. Esta etapa é essencial, uma vez que permite adaptar o Modelo ao contexto em que ele está a ser implementado.

A terceira etapa (implementação do Modelo SafeCare) diz respeito à intervenção, onde ocorrem as sessões de supervisão em grupo, que têm como objetivo promover a reflexão e a discussão sobre as necessidades em supervisão clínica identificadas pelos enfermeiros, assim como administrar formação sobre as variáveis definidas na etapa de diagnóstico de situação.

Por último, a quarta etapa (avaliação dos resultados) tem como objetivo avaliar a eficácia da implementação do Modelo SafeCare, voltando a aplicar-se o instrumento de avaliação do autocuidado utilizado na etapa 1.

O autocuidado é central na intervenção dos enfermeiros e assume-se como uma das áreas potenciadoras de ganhos em saúde, sendo reconhecido como um indicador de qualidade dos cuidados e como um critério de qualidade do exercício profissional ${ }^{(8-9)}$. De acordo com a Classificação Internacional para a Prática de Enfermagem (CIPE ${ }^{\circledR}$ ), o autocuidado é definido como "[...] atividade executada pelo próprio: tratar do que é necessário para se manter; manter-se operacional e lidar com as necessidades individuais básicas e íntimas e as atividades da vida diária” ${ }^{,(10: 35)}$, integrando, entre outras, tomar banho [higiene], alimentar-se, e virar-se [posicionar-se].

No contextual atual, importa que a avaliação da dependência se faça em relação a cada domínio de autocuidado, de forma a planearem-se cuidados individualizados e definir e implementar intervenções realistas e adequadas às necessidades dos pacientes ${ }^{(11)}$. Deste modo, a utilização de estratégias de supervisão clínica em enfermagem reveste-se de extrema importância, na medida em que, ao dotarem os enfermeiros de conhecimentos e capacidades que lhes permitam identificar os diagnósticos e as intervenções de enfermagem mais ajustados às necessidades dos pacientes, viabilizam uma prática de enfermagem mais significativa para eles, potenciando a sua recuperação de forma mais eficiente. Importa ainda realçar que a supervisão clínica em enfermagem é benéfica para o trabalho de enfermagem, na medida em que aumenta a satisfação profissional dos enfermeiros ${ }^{(12)}$ e potencializa o trabalho em equipe ${ }^{(2)}$.

Tomando por base informações dos instrumentos de avaliação de autocuidado preenchidos, os objetivos do estudo são: avaliar a concordância, relacionada ao grau de dependência no autocuidado, entre pesquisador, enfermeiros e prontuários antes e após implementação do Modelo SafeCare e avaliar as intervenções de enfermagem relativas ao autocuidado prescritas, antes e após implementação do Modelo SafeCare.

\section{Método}

Trata-se de estudo quantitativo, quase-experimental, de tipo pré-teste e pós-teste, sem grupo controle. A população em estudo são os pacientes internados em dois serviços de medicina num hospital do Norte de Portugal. A amostra, obtida por método de amostragem não probabilística por conveniência, integra 216 pacientes. Os pacientes selecionados pelos enfermeiros deveriam encontrar-se internados num dos dois serviços de medicina onde decorreu a investigação.

A recolha de dados realizou-se com recurso a um formulário - Instrumento de Avaliação do Autocuidado (IAC) - elaborado para o efeito e baseado no "Formulário de Avaliação da Dependência no Autocuidado" "(13). O formulário IAC foi criado, uma vez que se verificou a ausência de um instrumento que possibilitasse avaliar a concordância no grau de dependência e nas intervenções de enfermagem relativas ao autocuidado higiene, alimentar-se e posicionar-se, prescritas pelo pesquisador, enfermeiros e prontuários. Após a construção do formulário, foram efetuadas reuniões com Professores de 
Enfermagem, peritos em sistemas de informação e reconstrução de autonomia, que entenderam que o IAC poderia ser utilizado como instrumento de coleta de dados (validade de conteúdo). O formulário foi aplicado a 20 enfermeiros de serviços que não os selecionados para a implementação do Modelo SafeCare, que não propuseram alterações. Assim, o formulário contempla duas partes: a caracterização sociodemográfica e clínica dos pacientes; e a avaliação do grau de dependência e das intervenções de enfermagem referentes ao autocuidado higiene, alimentar-se e posicionar-se. O formulário integra ainda 156 itens. As respostas são assinaladas numa escala Likert de 4 pontos, em que 1 corresponde a "independente" e 4 a "dependente em grau elevado", apresentando um índice total de consistência interna adequado $(\alpha=0,99)$, avaliado pelo coeficiente alfa de Cronbach.

A aplicação do formulário realizou-se em dois momentos distintos: antes e após a implementação do Modelo SafeCare (pré-teste e pós-teste). O pré-teste transcorreu de outubro a dezembro de 2017, e o pós-teste realizou-se de janeiro a março de 2019. O procedimento de recolha de dados foi idêntico para o pré-teste e para o pós-teste.

Foi solicitado a cada enfermeiro que selecionasse dois pacientes, entre aqueles a quem estava a prestar cuidados nesse turno, para participar do estudo, e que preenchesse o formulário relativamente a esses mesmos pacientes.

Para cada paciente, foram preenchidos três formulários: o pesquisador preencheu, individualmente, o formulário para cada um dos pacientes; o enfermeiro preencheu, individualmente, o formulário para cada um dos pacientes; e o pesquisador preencheu também um formulário para cada paciente com a transcrição da informação registrada pelos enfermeiros no prontuário sobre os diagnósticos de enfermagem e as intervenções de enfermagem prescritas para o paciente no SClínico ${ }^{\circledR}$. O Sistema de Cuidados de Saúde Hospitalares (SClínico ${ }^{\circledR}$ ) é um sistema de informação evolutivo, presente em praticamente todos os hospitais de Portugal, sendo comum a todos os prestadores de cuidados de saúde (enfermeiros, médicos e outros profissionais de saúde) ${ }^{(14)}$. O SClínico ${ }^{\circledR}$ prevê a uniformização dos procedimentos dos prontuários, de forma a garantir a normalização da informação e a homogeneidade das práticas no Serviço Nacional de Saúde, o que se traduz num melhor apoio, assistência e acompanhamento ao paciente ${ }^{(14)}$.

Depois de preenchidos, os formulários foram emparelhados de forma que, para o mesmo paciente, se fizesse uma análise de concordância entre os dados obtidos nas três fontes (pesquisador, enfermeiros, prontuários).

A implementação do Modelo SafeCare ocorreu de janeiro a dezembro de 2018. Foi solicitado, a cada um dos enfermeiros, o preenchimento do contrato de supervisão clínica, a fim de ser formalmente assumida. Primeiramente, realizou-se formação em Supervisão Clínica para todos os enfermeiros, sendo abordadas, entre outras, as seguintes temáticas: modelos de supervisão clínica com ênfase no Modelo SafeCare, relação supervisiva, aprendizagem e desenvolvimento pessoal e profissional, qualidade de cuidados e segurança do cliente. Seguidamente, procedeu-se à identificação dos enfermeiros supervisores clínicos e enfermeiros supervisados. Em conjunto com os enfermeiros-chefes, foram constituídas as equipes de supervisão, numa razão de um enfermeiro supervisor clínico para oito a dez enfermeiros supervisados ${ }^{(4)}$. Foram calendarizadas as sessões de supervisão em grupo, com periocidade de uma sessão mensal por equipe de supervisão, com a duração de 90 minutos. A temática do autocuidado foi abordada com especial ênfase: nos autocuidados de higiene, alimentar-se, posicionar-se; nas necessidades em supervisão clínica identificadas pelos enfermeiros, como, por exemplo, comunicação e gestão de emoções; e nos prontuários $\left(\right.$ SClínico $\left.^{\circledR}\right)$. 
Fluxograma 1 - Etapas do Estudo

PRÉ-TESTE

outubro a dezembro de 2017

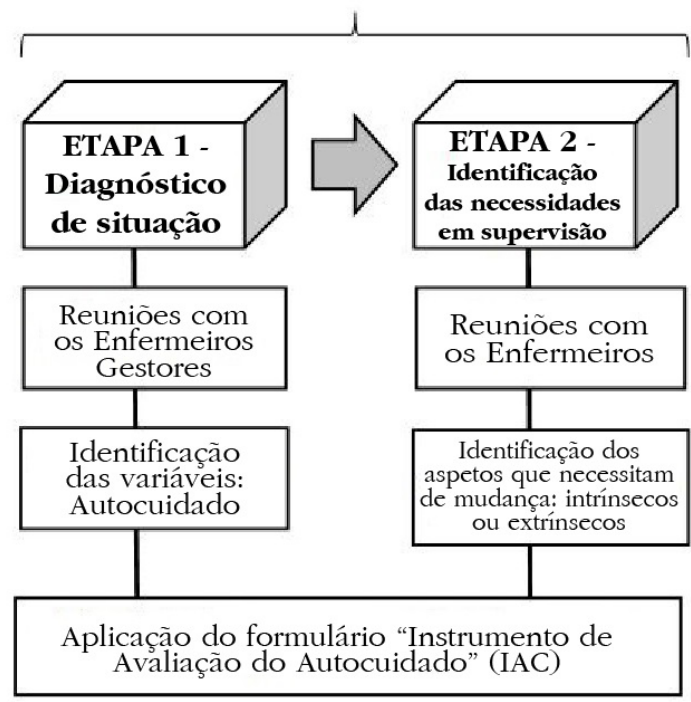

Fonte: Elaboração própria.

Para a análise dos dados, recorreu-se à estatística descritiva e ao coeficiente de concordância Kappa de Cohen, no programa estatístico Statistical Package for the Social Sciences (SPSS) versão 25.0, tendo sido considerado um nível de significância de 5\%. Para a interpretação do coeficiente de concordância Kappa de Cohen, teve-se em consideração os pontos de corte indicados por $\mathrm{McHugh}^{(15)}$ : valores entre 0 e 0,20 referem-se a um nível de concordância inexistente; valores entre 0,21 e 0,30 referem-se a um nível de concordância mínimo; valores entre 0,40 e 0,59 referem-se a um nível de concordância fraco; valores entre 0,60 e 0,79 referem-se a um nível de concordância moderado; valores entre 0,80 e 0,90 referem-se a um nível de concordância forte; e valores superiores a 0,90 referem-se a um nível de concordância quase perfeito.

A realização do estudo foi autorizada pelo Conselho de Administração e pela Comissão de Ética para a Saúde do hospital em 14 de julho de 2017 (nº 71/CE/JAS).

\section{Resultados}

A amostra foi constituída por 216 pacientes, sendo 123 homens $(56,9 \%)$ e 93 mulheres $(43,1 \%)$.
INTERVENÇÃO

PÓS-TESTE

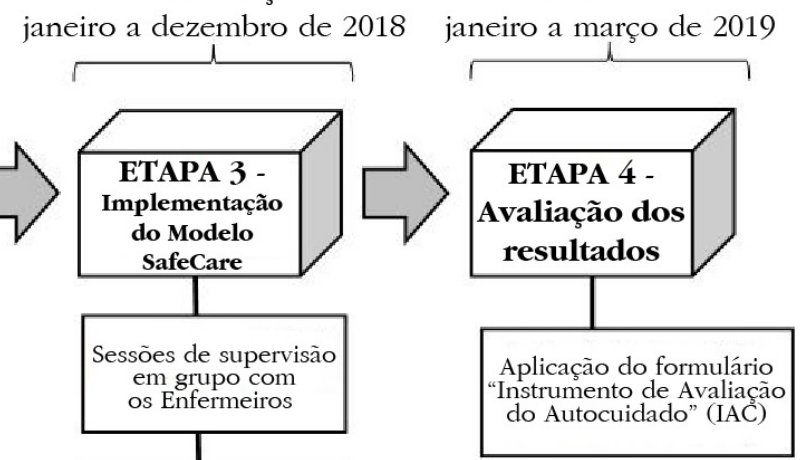

- Formação em supervisão clínica em enfermagem

- Constituição das equipes de supervisão

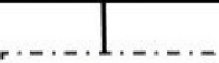

i Autocuidado i

.............
A idade dos participantes variou entre os 23 e os 96 anos, sendo a média de idades de aproximadamente 71 anos $(\mathrm{M}=71,44 ; \mathrm{DP}=14,16)$. Os pacientes apresentavam uma grande diversidade de diagnósticos médicos. As categorias de patologia com maior frequência de casos consistiram em doenças do sistema circulatório ( $n=99$; 45,83\%), doenças do sistema respiratório $(n=42 ; 19,44 \%)$, doenças do sistema neurológico $(n=19 ; 8,80 \%)$ e doenças do sistema urinário $(n=15 ; 6,94 \%)$.

Relativamente ao autocuidado higiene, verificou-se que existiu concordância entre as três observações no pré-teste e no pós-teste, mas, em ambos, a concordância foi superior entre os enfermeiros e os prontuários (pré-teste: $\mathrm{k}=0,72$; pós-teste: $\mathrm{k}=0,83$ ) e inferior entre o pesquisador e os prontuários (pré-teste: $\mathrm{k}=0,53$; pós-teste: $\mathrm{k}=0,68)$. A concordância entre $\mathrm{O}$ pesquisador e os enfermeiros no pré-teste $(\mathrm{k}=0,65)$ e no pós-teste $(\mathrm{k}=0,79)$ manteve-se moderada. Já a concordância entre o pesquisador e os prontuários aumentou de fraca no pré-teste $(\mathrm{k}=0,53)$ para moderada no pós-teste $(\mathrm{k}=0,68)$, assim como a concordância entre os enfermeiros e os prontuários, que aumentou de moderada no pré-teste $(\mathrm{k}=0,72)$ para forte no pós-teste $(\mathrm{k}=0,83)$. 
No que respeita ao autocuidado alimentar-se, verificou-se que existiu concordância entre as três observações no pré-teste e no pós-teste, mas, em ambos, a concordância foi inferior entre o pesquisador e os prontuários (pré-teste: $\mathrm{k}=0,57$; pós-teste: $\mathrm{k}=0,63$ ). A concordância entre o pesquisador e os enfermeiros aumentou de fraca no pré-teste $(\mathrm{k}=0,59)$ para moderada no pós-teste $(\mathrm{k}=0,63)$, acontecendo o mesmo com a concordância entre o pesquisador e os prontuários (pré-teste: k=0,57; pós-teste: $\mathrm{k}=0,63)$. A concordância entre os enfermeiros e os prontuários manteve-se moderada em ambos os momentos (pré-teste: $\mathrm{k}=0,71$; pós-teste: $\mathrm{k}=0,72$ ).
Por último, no autocuidado posicionar-se, verificou-se que existiu concordância entre as três observações no pré-teste e no pós-teste, mas, em ambos, a concordância foi superior entre os enfermeiros e os prontuários (pré-teste: $\mathrm{k}=0,74$; pós-teste: $\mathrm{k}=0,80)$. O grau de concordância entre as três fontes de obtenção de dados aumentou do pré-teste para o pós-teste.

A Tabela 1 apresenta os resultados relativos à concordância entre as três fontes de obtenção de dados (pesquisador, enfermeiros, prontuários) obtidos por meio do coeficiente de concordância Kappa de Cohen, relativamente ao grau de dependência no autocuidado dos pacientes no pré-teste e no pós-teste.

Tabela 1 - Concordância entre pesquisador, enfermeiros e prontuários na avaliação do grau de dependência dos pacientes no autocuidado (higiene, alimentar-se e posicionar-se) no pré-teste e no pós-teste. Porto, Portugal - 2017-2019. (N=216)

\begin{tabular}{|c|c|c|c|c|}
\hline \multirow{2}{*}{\multicolumn{2}{|c|}{$\begin{array}{l}\text { Grau de } \\
\text { dependência } \\
\text { dos pacientes }\end{array}$}} & \multicolumn{3}{|c|}{ Kappa de Cohen } \\
\hline & & Pesquisador-Enfermeiros & Pesquisador-Prontuários & Enfermeiros-Prontuários \\
\hline \multirow{3}{*}{ 离 } & Higiene & $0,65^{*}$ & $0,53^{*}$ & $0,72 *$ \\
\hline & Alimentar-se & $0,59 *$ & $0,57^{*}$ & $0,71^{*}$ \\
\hline & Posicionar-se & $0,56^{*}$ & $0,55^{*}$ & $0,74^{*}$ \\
\hline \multirow{3}{*}{ 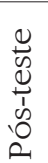 } & Higiene & $0,79 *$ & $0,68^{*}$ & $0,83^{*}$ \\
\hline & Alimentar-se & $0,73^{*}$ & $0,63^{*}$ & $0,72 *$ \\
\hline & Posicionar-se & $0,79 *$ & $0,80^{*}$ & $0,80 *$ \\
\hline
\end{tabular}

Fonte: Elaboração própria.

$* \mathrm{p}<0,05$

Relativamente ao autocuidado higiene, verificou-se que, para a intervenção de enfermagem "incentivar o autocuidado: higiene", só existiu concordância entre os enfermeiros e os prontuários $(\mathrm{k}=0,54)$ no pré-teste, enquanto, no pós-teste, existiu concordância entre as três observações. A concordância entre os enfermeiros e os prontuários aumentou de fraca no pré-teste $(\mathrm{k}=0,54)$ para muito forte no pós-teste $(\mathrm{k}=0,92)$. Na intervenção de enfermagem "avaliar autocuidado: higiene", verificou-se que existiu concordância entre as três observações no pré-teste e no pós-teste, mas, em ambos, a concordância foi inferior entre o pesquisador e os prontuários (pré-teste: $\mathrm{k}=0,40$; pós-teste: $\mathrm{k}=0,52)$. A concordância entre o pesquisador e os enfermeiros manteve-se moderada do pré-teste $(\mathrm{k}=0,70)$ para o pós-teste $(\mathrm{k}=0,78)$ e, entre o pesquisador e os prontuários, manteve-se fraca do pré-teste $(\mathrm{k}=0,40)$ para o pós-teste $(k=0,52)$. A concordância entre enfermeiros e prontuários aumentou de fraca no pré-teste $(\mathrm{k}=0,53)$ para moderada no pós-teste $(\mathrm{k}=0,79)$.

No que respeita ao autocuidado alimentar-se, verificou-se que, para a intervenção de enfermagem "vigiar refeição", existiu concordância entre as três observações no pré-teste e no pós-teste, mas, em ambos, a concordância foi superior entre o pesquisador e os enfermeiros (pré-teste: $\mathrm{k}=0,55$; pós-teste: $\mathrm{k}=0,72$ ), e inferior entre o pesquisador e os prontuários (pré-teste: $\mathrm{k}=0,26$; pós-teste: $\mathrm{k}=0,38$ ). A concordância entre o pesquisador e os enfermeiros aumentou de 
fraca no pré-teste $(\mathrm{k}=0,55)$ para moderada no pós-teste $(\mathrm{k}=0,72)$, acontecendo o mesmo na concordância entre os enfermeiros e os prontuários (pré-teste: k=0,46; pós-teste: k=0,61). Já a concordância entre o pesquisador e os prontuários manteve-se mínima antes e após a intervenção (pré-teste: k=0,26; pós-teste: $\mathrm{k}=0,38$ ). Na intervenção de enfermagem "avaliar o alimentar-se", verificou-se que existiu concordância entre as três observações no pré-teste e no pós-teste, mas, em ambos, a concordância foi superior entre o pesquisador e os enfermeiros (pré-teste: $\mathrm{k}=0,63$; pós-teste: $\mathrm{k}=0,71$ ), e inferior entre o pesquisador e os prontuários (pré-teste: k=0,28; pós-teste: $\mathrm{k}=0,37$ ). A concordância entre o pesquisador e os enfermeiros manteve-se moderada entre o pré-teste $(\mathrm{k}=0,63)$ e o pós-teste $(\mathrm{k}=0,71)$, assim como entre os enfermeiros e os prontuários (pré-teste: k=0,62; pós-teste: k=0,62). A concordância entre o pesquisador e os prontuários manteve-se mínima entre o pré-teste $(\mathrm{k}=0,28)$ e o pós-teste $(\mathrm{k}=0,37)$.

Por último, no autocuidado posicionar-se, verificou-se que, para a intervenção de enfermagem "incentivar a posicionar-se", só existiu concordância entre o pesquisador e os enfermeiros $(\mathrm{k}=0,62)$ e entre os enfermeiros e os prontuários $(\mathrm{k}=0,44)$ no pré-teste, enquanto, no pós-teste, existiu concordância entre as três observações. A concordância entre o pesquisador e os enfermeiros diminuiu de moderada no pré-teste $(\mathrm{k}=0,62)$ para fraca no pós-teste $(k=0,59)$, enquanto a concordância entre os enfermeiros e os prontuários aumentou de fraca no pré-teste $(\mathrm{k}=0,44)$ para forte no pós-teste $(k=0,80)$. Na intervenção de enfermagem "avaliar o posicionar-se", verificou-se que existiu concordância entre as três observações no pré-teste e no pós-teste, mas, em ambos, a concordância foi inferior entre o pesquisador e os prontuários (pré-teste: $\mathrm{k}=0,34$; pós-teste: $\mathrm{k}=0,58$ ). A concordância aumentou nas três observações do pré-teste para o pós-teste.

A Tabela 2 apresenta os resultados relativos à concordância entre as três fontes de obtenção de dados (pesquisador, enfermeiros, prontuários) obtidos pelo coeficiente de concordância Kappa de Cohen, no que concerne às intervenções de enfermagem prescritas no pré-teste e no pós-teste. É de salientar que não foi possível avaliar a concordância em todas as intervenções de enfermagem incluídas no formulário, devido à reduzida dimensão amostral (número de respostas obtidas) para algumas dessas intervenções.

Tabela 2 - Concordância entre pesquisador, enfermeiros, e prontuários na avaliação das intervenções de enfermagem relativas ao autocuidado (higiene, alimentar-se e posicionar-se) no pré-teste e no pósteste. Porto, Portugal - 2017-2019. (N=216)

\begin{tabular}{|c|c|c|c|c|c|}
\hline \multirow{2}{*}{\multicolumn{3}{|c|}{$\begin{array}{l}\text { Intervenções de enfermagem relativas ao } \\
\text { autocuidado }\end{array}$}} & \multicolumn{3}{|c|}{ Kappa de Cohen } \\
\hline & & & \multirow{2}{*}{$\begin{array}{c}\text { Pesquisador- } \\
\text {-Enfermeiros }\end{array}$} & \multirow{2}{*}{$\begin{array}{c}\text { Pesquisador- } \\
\text {-Prontuários } \\
0,21\end{array}$} & \multirow{3}{*}{$\begin{array}{c}\text { Enfermeiros- } \\
\text {-Prontuários } \\
0,54^{*}\end{array}$} \\
\hline \multirow{6}{*}{ 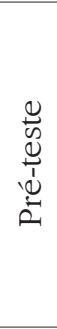 } & Higiene & $\begin{array}{l}\text { Incentivar o autocuidado: } \\
\text { higiene }\end{array}$ & & & \\
\hline & & Avaliar autocuidado: higiene & $0,70^{*}$ & $0,40^{*}$ & \\
\hline & Alimentar-se & Vigiar refeição & $0,55^{*}$ & $0,26^{*}$ & $0,46 *$ \\
\hline & & Avaliar o alimentar-se & $0,63^{*}$ & $0,28^{*}$ & $0,62^{*}$ \\
\hline & Posicionar-se & Incentivar a posicionar-se & $0,62 *$ & 0,27 & $0,44^{*}$ \\
\hline & & Avaliar o posicionar-se & $0,59 *$ & $0,34^{*}$ & $0,43^{*}$ \\
\hline \multirow{6}{*}{ 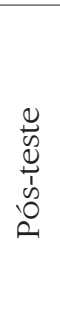 } & Higiene & $\begin{array}{l}\text { Incentivar o autocuidado: } \\
\text { higiene }\end{array}$ & $0,85^{*}$ & $0,79 *$ & $0,92 *$ \\
\hline & & Avaliar autocuidado: higiene & $0,78^{*}$ & $0,52^{*}$ & $0,79 *$ \\
\hline & Alimentar-se & Vigiar refeição & $0,72^{*}$ & $0,38^{*}$ & $0,61^{*}$ \\
\hline & & Avaliar o alimentar-se & $0,71^{*}$ & $0,37^{*}$ & $0,62^{*}$ \\
\hline & Posicionar-se & Incentivar a posicionar-se & $0,59 *$ & $0,41^{*}$ & $0,80^{*}$ \\
\hline & & Avaliar o posicionar-se & $0,78^{*}$ & $0,58^{*}$ & $0,79 *$ \\
\hline
\end{tabular}

Fonte: Elaboração própria.

$* \mathrm{p}<0,05$ 


\section{Discussão}

Relativamente ao primeiro objetivo deste estudo, verificou-se um aumento da concordância entre as três observações relativo ao grau de dependência dos pacientes em todos os autocuidados (à exceção entre o pesquisador e os enfermeiros no autocuidado higiene, e entre os enfermeiros e os prontuários no autocuidado alimentar-se, em que a concordância manteve-se moderada em ambos os momentos). Os resultados obtidos são semelhantes aos encontrados em outro estudo(16), o qual confirma que, após a implementação de um modelo de supervisão clínica com 111 pacientes de um hospital do Norte de Portugal, existia maior concordância entre o grau de dependência atribuído pelos enfermeiros especialistas e pelos enfermeiros generalistas.

Esses resultados comprovam que a implementação do Modelo SafeCare foi eficaz, uma vez que os enfermeiros começaram a identificar o grau de dependência dos pacientes nos autocuidados avaliados de forma mais concordante com o pesquisador, que é considerado o padrão ouro (gold standard). Este resultado pode indicar que as estratégias de supervisão clínica utilizadas no Modelo SafeCare foram também eficazes para promover uma reflexão crítica e, consequentemente, uma alteração das práticas dos enfermeiros relacionadas com o autocuidado.

No que respeita ao segundo objetivo deste estudo, verificou-se que nem sempre existiu concordância significativa entre as três fontes de obtenção de dados antes da intervenção. De fato, as únicas fontes de obtenção de dados que apresentaram sempre concordância no pré-teste foram os enfermeiros e os prontuários. Por sua vez, no pós-teste, obteve-se concordância significativa entre as três fontes de obtenção de dados e em todas as intervenções de enfermagem. Estes resultados são semelhantes aos encontrados em outro estudo $^{(16)}$, que verificou aumento no número de correspondências na seleção das intervenções pelos enfermeiros especialistas e enfermeiros generalistas após a implementação de um modelo de supervisão clínica.
No presente estudo, observou-se ainda, no pós-teste, aumento da concordância entre o pesquisador e os enfermeiros em todas as intervenções de enfermagem em que se registrou uma concordância inferior à moderada no pré-teste, à exceção da intervenção de enfermagem "incentivar a posicionar-se", na qual a concordância entre estas duas fontes de obtenção de dados diminuíram após a intervenção. Este resultado indica a necessidade de sensibilizar ainda mais os enfermeiros, para solicitarem a participação ativa dos pacientes quando do seu posicionamento.

Assim, poder-se-á afirmar que houve, na sua maioria, um aumento da concordância das três fontes de obtenção de dados na identificação do grau de dependência no autocuidado e na seleção das intervenções de enfermagem prescritas após a implementação do Modelo SafeCare, pelo que se poderá concluir que possibilitou que os enfermeiros refletissem criticamente sobre o planeamento dos seus cuidados, identificassem aspetos que necessitavam de mudança e alterassem a sua forma de concetualizar os cuidados, tornando-os mais significativos para os pacientes, uma vez que se direcionam para as reais necessidades deles.

O presente estudo apresenta algumas limitações que restringem e dificultam a interpretação e extrapolação dos resultados. A técnica de amostragem e o fato de a recolha de dados ter sido realizada apenas em dois serviços de medicina do mesmo hospital constituem uma limitação à generalização dos resultados. Além disso, a ausência de um grupo controle também é considerada uma limitação, uma vez que o grupo controle permite estabelecer uma inferência de causalidade por meio de comparação, assegurando que as diferenças observadas entre o grupo experimental e o grupo controle possam ser atribuídas à intervenção realizada ${ }^{(17)}$. Outra limitação prende-se ao fato de o formulário IAC ser de autopreenchimento, o que poderá levar a alguns enviesamentos originados pela desejabilidade social, respostas aleatórias, entre outros. É também de salientar que não foi possível avaliar a concordância em todas as intervenções de enfermagem incluídas no formulário, devido 
à reduzida dimensão amostral (número de respostas obtidas) para algumas dessas intervenções.

Não obstante, este estudo engloba aspetos importantes e inovadores. De fato, a evidência sugere que, apesar de existirem modelos de supervisão clínica com impacto positivo nas instituições de saúde $^{(7)}$, no trabalho dos enfermeiros ${ }^{(1)}$ e nos cuidados prestados aos pacientes ${ }^{(18)}$, a sua operacionalização não é clara, tornando-se difícil a sua replicação em outros contextos ${ }^{(2,19)}$. Ao abordar a operacionalização e a avaliação do impacto da implementação do Modelo SafeCare no âmbito do autocuidado, este estudo sobressai pela sua originalidade. Além disso, existem poucos estudos quantitativos sobre a temática da supervisão clínica em enfermagem $^{(2)}$. O presente estudo pretendeu dar resposta a esta lacuna, constituindo-se, assim, em importante contributo para o avanço do conhecimento científico na área da supervisão clínica em Enfermagem. Em suma, o Modelo SafeCare surge como uma ferramenta que permite uma aproximação das competências dos enfermeiros que possibilitam melhorias no seu exercício profissional e na identificação de áreas de intervenção, nomeadamente no âmbito da identificação de diagnósticos e de intervenções de enfermagem a prescrever aos pacientes.

\section{Conclusão}

Tendo em consideração os objetivos definidos para esta investigação, constatou-se que a implementação do Modelo SafeCare permitiu aumentar a concordância na identificação do grau de dependência no autocuidado, atribuídos pelo pesquisador, enfermeiros e nos prontuários, assim como nas intervenções de enfermagem, com exceção da intervenção "incentivar a posicionar-se". O Modelo SafeCare, ao utilizar estratégias de supervisão clínica adequadas ao contexto da sua implementação, possibilitou o desenvolvimento de competências nos enfermeiros para avaliar e intervir no autocuidado, visíveis nos resultados deste estudo. Em suma, o Modelo SafeCare, ao permitir que os cuidados de enfermagem sejam mais adequados às reais necessidades dos pacientes, revestem-nos de uma qualidade efetiva, podendo traduzir-se em ganhos significativos na prática clínica.

\section{Colaborações:}

1 - concepção, projeto, análise e interpretação dos dados: Inês Rocha, António Luís Carvalho, Cristina Barroso Pinto, Agostinho Rodrigues e Vânia Rocha;

2 - redação do artigo e revisão crítica relevante do conteúdo intelectual: Inês Rocha, António Luís Carvalho, Cristina Barroso Pinto e Vânia Rocha;

3 - aprovação final da versão a ser publicada: Inês Rocha, António Luís Carvalho, Cristina Barroso Pinto, Agostinho Rodrigues e Vânia Rocha.

\section{Referências}

1. Buus N, Lynch L, Gonge H. Developing and implementing 'meta-supervision' for mental health nursing staff supervisees: Opportunities and challenges. Cogn Behav Therap. 2016;9(e22): 323-34. DOI: 10.1017/S1754470X15000434

2. Cutcliffe J, Sloan G, Bashaw M. A systematic review of clinical supervision evaluation studies in nursing. Int J Ment Health Nurs. 2018;27(5): 1344-63. DOI: 10.1111/inm.12443

3. Ordem dos Enfermeiros. Caderno temático - Modelo de desenvolvimento profissional: fundamentos, processos e instrumentos para a operacionalização do Sistema de Certificação de Competências. Lisboa (PT); 2010.

4. Barroso C, Carvalho L, Augusto C, Teixeira L, Teixeira A, Rocha I. Implementação de um modelo de supervisão clínica em enfermagem - modelo SAFECARE. Rev Rol enferm [Internet]. 2020 [cited 2021 Jan 15];43(supl 1):50-8. Available from: https://pesquisa.bvsalud.org/portal/resource/pt/ ibc-193162

5. Augusto M, Oliveira K, Carvalho A, Pinto C, Teixeira A, Teixeira L. Impact of a model of clinical supervision over the emotional intelligence capacities of nurses. Rev Rene. 2021;22:e60279. DOI: $10.15253 / 2175-6783.20212260279$

6. Carvalho A, Barroso C, Pereira M, Teixeira A, Pinho F, Osório M. Implementação de um modelo 
de supervisão clínica em enfermagem. Porto: Uniarte Gráfica; 2019.

7. Snowdon D, Leggat S, Taylor N. Does clinical supervision of healthcare professionals improve effectiveness of care and patient experience? A systematic review. BMC Health Serv Res. 2017;17(1):786. DOI: 10.1186/s12913-017-2739-5

8. Ordem dos Enfermeiros. Padrões de qualidade dos cuidados de enfermagem: enquadramento conceptual e enunciados descritivos. Lisboa (PT); 2012.

9. Teixeira S, Carvalho A, Cruz S. Self-care assessement as an indicator for clinical supervision in nursing. Rev Rene. 2016;17(3): 356-62. DOI: 10.15253/2175-6783.2016000300008

10. International Council of Nurses. Classificação Internacional para a Prática de Enfermagem (CIPE): versão 2.0 [Internet]. Lisboa: Ordem dos Enfermeiros; 2011 [cited 2020 Dec 27]. Available from: http://www.ordemenfermeiros.pt/browser CIPE/BrowserCIPE.aspx

11. Petronilho F, Pereira C, Magalhães A, Carvalho D, Oliveira J, Castro P, et al. Evolution of self-care dependent individuals admitted to the National Network for Integrated Continuous Care. Rev Enf Ref. 2017;série IV(14):39-48. DOI: 10.12707/ RIV17027

12. Boamah S, Read E, Laschinger H. Factors influencing new graduate nurse burnout development, job satisfaction and patient care quality: a time-lagged study. J Adv Nurs. 2017;73(5):1182-95. DOI: 10.1111/jan.13215

13. Pereira S, Martins T, Machado PP. Formulário de avaliação de dependência no autocuidado. In: Jornadas Internacionais de Enfermagem Comunitária, 2016, Porto (PT). Livro de comunicações [Internet]. Porto: Escola Superior de Enfermagem do Porto; 2016. p. 96-103 [cited 2020 Dec 2]. Available from: https://comum.rcaap.pt/ bitstream/10400.26/31762/1/2016_Jornadas96-103.pdf
14. Bailas CMO. Impacto do uso de sistemas de informação informatizados na carga global de trabalho dos enfermeiros [dissertação]. [Internet]. Porto (PT): Escola Superior de Enfermagem do Porto; 2016 [cited 2020 Dec 14]. Available from: https://comum.rcaap.pt/ bitstream/10400.26/17135/1/Tese\%20Marisa\%20 Bailas.pdf

15. McHugh M. Interrater reliability: the kappa statistic. Biochem Med [Internet]. 2012 [cited 2021 Jan 21];22(3):276-82. Available from: https:// pubmed.ncbi.nlm.nih.gov/23092060/

16. Ferreira A. Autocuidado como indicador de qualidade e segurança dos cuidados: contributos da supervisão clínica em enfermagem [dissertação]. [Internet]. Porto (PT): Escola Superior de Enfermagem do Porto; 2015 [cited 2020 Dec 23]. Available from: https:// comum.rcaap.pt/bitstream/10400.26/9301/1/ Disserta\%c3\%a7\%c3\%a3o\%20Mestrado\%20-\%20 S\%c3\%adlvia\%20Teixeira.pdf

17. Dutra H, Reis V. Desenhos de estudos experimentais e quase-experimentais: definições e desafios na pesquisa em enfermagem. Rev Enf UFPE. 2016;10(6):2230-41. DOI: 10.5205/ reuol.9199-80250-1-SM1006201639

18. Snowdon D, Hau R, Leggat S, Taylor N. Does clinical supervision of health professionals improve patient safety? A systematic review and meta-analysis. Int $\mathrm{J}$ Qual Health Care. 2016;28(4):447-55. DOI: 10.1093/intqhc/mzw059

19. Pollock A, Campbell P, Deery R, Fleming M, Rankin J, Sloan G, et al. A systematic review of evidence relating to clinical supervision for nurses, midwives and allied health professionals. J Adv Nurs. 2017;73(8):1825-37. DOI: 10.1111/ jan. 13253

Recebido: 5 de fevereiro de 2021

Aprovado: 30 de maio de 2021

Publicado: 22 de julho de 2021

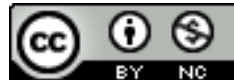

A Revista Baiana de Enfermagem utiliza a Licença Creative Commons - Atribuição-NãoComercial 4.0 Internacional. https://creativecommons.org/licenses/by-nc/4.0/ Este artigo é de acesso aberto distribuído sob os termos da Licença Creative Commons (CC BY-NC). Esta licença permite que outros remixem, adaptem e criem a partir do seu trabalho para fins não comerciais. Embora os novos trabalhos tenham de lhe atribuir o devido crédito e não possam ser usados para fins comerciais, os usuários não têm de licenciar esses trabalhos derivados sob os mesmos termos. 